\title{
Cloning, expression and purification of botulinum neurotoxin type A heavy chain - crystallographic evidence for a putative tetrameric pore
}

\section{Damodharan Lakshminarasimhan, Desigan Kumaran and Rakhi Agarwal}

Biology Department,

Brookhaven National Laboratory,

Upton, NY 11973, USA

E-mail: laksdhamu@gmail.com

E-mail: kumaran@bnl.gov

E-mail: agarwal@bnl.gov

\section{Bal Ram Singh}

University of Massachusetts Dartmouth, Dartmouth, MA 02747, USA

E-mail: bsingh@umassd.edu

\section{Subramanyam Swaminathan*}

Biology Department,

Brookhaven National Laboratory,

Upton, NY 11973, USA

E-mail: swami@bnl.gov

*Corresponding author

\begin{abstract}
Clostridium botulinum produces seven antigenically distinct serotypes of botulinum neurotoxin (BoNTs, A-G) the most potent toxins to humans. They cause paralysis at less than picomolar concentration by blocking neurotransmitter release. BoNT consists of a heavy chain and a light chain. The C-terminal half of the heavy chain allows the toxin to bind to the presynaptic membrane while the N-terminal half helps in forming a channel in the endosomal membrane to allow the light chain to escape into the cytosol. While the binding and catalytic mechanisms are well understood now, the details of translocation still remain a mystery. A full length BoNT/A heavy chain (BAHC) has been cloned, over expressed and purified from inclusion bodies by solubilising with a detergent. Preliminary crystallographic results show that BAHC forms a tetramer in the crystal lending experimental support for tetrameric pore formation for the light chain to pass through the endosomal membrane.
\end{abstract}

Keywords: botulinum neurotoxin; BoNT; translocation; oligomerisation; pore formation; heavy chain; HC; auto induction; inclusion bodies. 
Reference to this paper should be made as follows: Lakshminarasimhan, D., Kumaran, D., Agarwal, R., Singh, B.R. and Swaminathan, S. (2012) 'Cloning, expression and purification of botulinum neurotoxin type A heavy chain - crystallographic evidence for a putative tetrameric pore', The Botulinum J., Vol. 2, No. 2, pp.135-149.

Biographical notes: Damodharan Lakshminarasimhan is currently a Postdoc at University of Massachusetts at Lowell, MA. He is a Structural Biologist interested in studying structure - function relationships in proteins, especially toxins using $\mathrm{x}$-ray crystallography. He obtained his $\mathrm{PhD}$ from the University of Madras, India and post-doctoral training at Brookhaven National Laboratory, USA.

Desigan Kumaran is a Biophysicist at the Brookhaven National Laboratory (BNL) where he is carrying out structural biology research more specifically towards the drug development for botulinum neurotoxins and structural genomics project. He made very important contributions which have had a major impact in the biodefense and protein structure initiative projects. $\mathrm{He}$ completed his doctoral degree from the University of Madras, India and received post-doctoral training at BNL.

Rakhi Agarwal is an Associate-Biophysicist at the Biology Department, Brookhaven National Laboratory, New York, USA. She is an expert in biochemistry and structural biology (x-ray crystallography). She has made significant contributions on structure and function of various Clostridium botulinum neurotoxins and PSI-biology proteins. She is working on structure-based drug discovery for botulinum neurotoxin type $\mathrm{E}$ and $\mathrm{F}$.

Bal Ram Singh is a Professor of Chemistry and Biochemistry at the University of Massachusetts Dartmouth, and the founding Director of the National Botulinum Research Center at UMass Dartmouth, which has over three dozen national and international botulinum researchers associated with it. He has been working with structure and functions of botulinum neurotoxins since 1987, and is a leading scientist in the field. He received his $\mathrm{PhD}$ in Chemistry from Texas Tech University, and post-doctoral training at University of Wisconsin Madison.

Subramanyam Swaminathan is a Senior Scientist at the Biology Department, Brookhaven National Laboratory. He is a Macromolecular Crystallographer and Structural Biologist interested in structure-function relationships in proteins, especially botulinum neurotoxins. He is working on structure-based drug discovery for botulinum neurotoxins.

\section{Introduction}

Clostridium botulimum produces botulinum neurotoxins (BoNTs), the most potent toxins to humans. There are seven serologically distinct BoNTs (BoNT A-G). BoNTs are produced as a single polypeptide chain $(150 \mathrm{kDa})$ and cleaved exogenously or endogenously into a $100 \mathrm{kDa}$ heavy chain (HC) and a $50 \mathrm{kDa}$ light chain (LC) linked through a disulfide bond (Lacy and Stevens, 1999). BoNTs function via three steps, extra cellular binding through gangliosides and specific protein receptors at the presynaptic 
membrane, internalisation via energy mediated endocytosis, and endosomal membrane translocation of the LC into the cytosol. Once in the cytosol, the LC cleaves one of the three proteins of the SNARE complex and blocks the neurotransmitter release leading to flaccid paralysis (Simpson, 1986, 1989).

Neuronal cell surfaces contain low affinity gangliosides and high specificity protein receptors for BoNTs. Accordingly, a double receptor model has been proposed (Chai et al., 2006; Montecucco, 1986). In the absence of gangliosides the clostridial neurotoxins do not penetrate the cell (Yowler et al., 2002). Earlier reports show that BoNT/A and BoNT/B have single ganglioside binding site. Also, it is known that the translocation domain is able to form channels in cell membranes (Sheridan, 1998) and artificial bilayers (Blaustein et al., 1987; Donovan and Middlebrook, 1986; Hoch et al., 1985).

Crystal structures of BoNT/A and its catalytic and binding domains are available (Fu et al., 2009; Kumaran et al., 2008; Lacy et al., 1998; Pless et al., 2001; Segelke et al., 2004; Stenmark et al., 2008). While the binding and catalytic mechanisms are understood fairly well, the details about the translocation mechanism are only now slowly emerging (Fischer and Montal, 2007b; Fischer et al., 2008, 2009; Montal, 2009; Montal et al., 1990). The essential mechanism underlying the LC translocation through the endosomal membrane can be better understood with detailed information of the three-dimensional structure of the BoNT HC that forms the translocation channel. So far, structural information has been limited to low resolution electron microscopy for BoNT/B (Schmid et al., 1993), channel current studies, substrate proteolysis experiments (Koriazova and Montal, 2003) and single molecule detection of an intermediate during BoNT translocation across membranes (Fischer and Montal, 2007a). Recent fluorescence-labelled dextran studies show that the width of the membrane channel is at the most $24.2 \AA$ (Parikh and Singh, 2007). Since the dimension of the LC is $\sim 50 \AA$, it is not possible for it to penetrate the endosomal membrane without unfolding. Accordingly, it is suggested that it unfolds while passing through the channel (or before) and refolds once it is in the cytosol and is ready for proteolytic attack of its substrates. Since the HC alone is involved in translocation and formation of channel, we have chosen the BoNT/A heavy chain (hereafter BAHC) to study the pore/channel formation of these neurotoxins by X-ray diffraction. Lipid bilayer studies and channel current studies have shown that the HC forms a channel. The BAHC protein was expressed in $E$. coli as inclusion bodies and solubilised with anionic detergent LSN. Crystals obtained in the presence of detergent diffracted to better than $6.0 \AA$ and a self-rotation Patterson map showed a four-fold symmetry indicating a tetrameric association. Hence, the present findings suggest possible tetrameric state of BAHC in the presence of a detergent partly mimicking the lipid environment.

\section{Materials and methods}

\subsection{Primer design, polymerase chain reaction and gene construction}

\subsubsection{DNA preparation}

Genomic DNA was prepared as described previously (Singh et al., 1996). Clostridium botulinum type A (strain Hall), was inoculated in $10 \mathrm{ml}$ of toxin production media (2\% 
$\mathrm{N}-\mathrm{Z}$ amine, $0.5 \%$ yeast extract, $0.6 \%$ yeast paste for type A C. botulinum) and incubated at $37^{\circ} \mathrm{C}$ for $24 \mathrm{~h}$. High molecular weight total cellular DNA was isolated according to a previously reported method (Szabo et al., 1993). Briefly, DNA was recovered from alkaline cell lysis by centrifuging vegetative cells, washing twice with $1 \times$ SSC (sodium citrate, sodium chloride), resuspending in $1 \mathrm{M} \mathrm{NaOH}$ and heating at $65^{\circ} \mathrm{C}$ for $5 \mathrm{~min}$. Following centrifugation, the supernatants were collected and DNA was precipitated with isopropanol and $0.3 \mathrm{M}$ sodium acetate, $\mathrm{pH}$ 7.0. The DNA was pelleted, resuspended in water and purified using a DNA binding matrix (Prep-a-Gene, BioRad, Hercules, CA) equal to $25 \mathrm{ml}$ binding matrix/20 mg DNA. A quantity of binding buffer (6 M sodium perchlorate, $50 \mathrm{mM}$ Tris-HC1, $\mathrm{pH}$ 8.0, $10 \mathrm{mM}$ EDTA) equal to three times the combined volumes of Prep-a-Gene matrix suspension and DNA solution was incubated at room temperature for $10 \mathrm{~min}$ and centrifuged. The pellet was rinsed twice with binding buffer. After centrifugation the matrix-DNA was washed three times with $20 \mathrm{mM}$ Tris-HCl, pH 7.5, $2 \mathrm{mM}$ EDTA, 50\% ethanol. The bound DNA was eluted in $10 \mathrm{mM}$ Tris- $\mathrm{HCl} \mathrm{pH} 8.0,1 \mathrm{mM}$ EDTA, by incubating at $37^{\circ} \mathrm{C}$ to $50^{\circ} \mathrm{C}$ for $5 \mathrm{~min}$ followed by centrifugation. The DNA containing supernatant was stored at $4^{\circ} \mathrm{C}$.

\subsubsection{Gene construction}

Polymerase chain reaction (PCR) was performed with the primers 5'-GGGCCCCCATAT GGCATTAAATGATTTATGTATC-3' and 5'-GGGCCCCGGATCCTTACAGTGGCC TTTCTTCCCA-3' to generate a gene encoding the entire HC from BoNT/A according to an established procedure ( $\mathrm{Li}$ and Singh, 1999). Chromosomal DNA (50-100 ng) from C. botulinum (strain Hall) was used as template. An NdeI and a BamHI restriction site was incorporated into the 5' end of the forward sequence and reverse sequence primers, respectively. PCR reactions were carried out in a total volume of $50 \mu \mathrm{l}$ containing $10 \mathrm{mM}$ Tris/ $\mathrm{HCl}, \mathrm{pH} 8.3,50 \mathrm{mM} \mathrm{KCl}, 4 \mathrm{mM} \mathrm{MgCl} 2,0.1 \% \mathrm{BSA}, 100 \mu \mathrm{M}$ concentration of each dNTP, $10 \mathrm{mM} \beta$-mercaptoethanol ( $\beta-\mathrm{Me}), 25$ pmol of each primer, 2.5 units of DNA polymerase (New England Biolabs). The reaction mixtures were denatured for $2 \mathrm{~min}$ at $93^{\circ} \mathrm{C}$ and then subjected to 30 consecutive cycles consisting of denaturation $\left(1 \mathrm{~min}\right.$ at $\left.90^{\circ} \mathrm{C}\right)$, annealing $\left(2 \min\right.$ at $50^{\circ} \mathrm{C}$ ) and polymerisation $\left(3 \mathrm{~min}\right.$ at $73^{\circ} \mathrm{C}$ ). After amplification, the DNA fragments were cleaved with NdeI and BamHI and then electrophoresed on a 1.0\% agarose gel. The NdeI-BamHI digested fragment was purified from the agarose gel using QIAGEN columns (QIAGEN Inc. Valencia, CA) and then ligated to the NdeI/BamHI digested vector pET-15b. A typical ligation reaction mixture contained $40 \mathrm{ng}$ vector and $200 \mathrm{ng}$ insert in a volume of $20 \mu \mathrm{l}$ of water. $3 \mu \mathrm{l}$ of $10 \times$ ligase buffer and $1 \mu \mathrm{l}$ of T4 ligase was used. After overnight incubation at $16^{\circ} \mathrm{C}$, the ligation product was transformed into competent $E$. coli strain XL1-Blu and grew in Luria broth supplemented with ampicillin $(100 \mu \mathrm{g} / \mathrm{ml})$.

Clones were screened by digestion of a miniprep (Promega Corp., Madison, WI) plasmid DNA with BamHI and NdeI. Confirmation that the amplified fragment encoded BAHC was obtained by DNA sequencing of a representative plasmid recombinant (designated pET-HCA). DNA sequencing was performed by the dideoxy chain termination method. 


\subsection{Expression, solubilisation and purification of the recombinant BoNT/A HC}

\subsubsection{Expression}

Five millilitres of minimal medium for cell growth (MDG) medium containing $50 \mu \mathrm{g} / \mathrm{ml}$ ampicillin and $25 \mu \mathrm{g} / \mathrm{ml}$ chloramphenicol was inoculated with the freezer stock of BL21 (DE3) cells containing pET-HCA. After overnight growth at $37^{\circ} \mathrm{C}, 500 \mu \mathrm{l}$ of this culture was used to inoculate $500 \mathrm{ml}$ of ZYP 5052 [auto induction medium (Studier, 2005)] with ampicillin and chloramphenicol in a $2 \mathrm{~L}$ flask and the cells were grown at $37^{\circ} \mathrm{C}$ with shaking at $300 \mathrm{rpm}$ until the $\mathrm{A}_{600}$ reached 0.8 . At this point, the cells were incubated at $20^{\circ} \mathrm{C}$ for an additional $18 \mathrm{~h}$ shaking at $200 \mathrm{rpm}$. The induced cells were harvested by centrifugation at $6,000 \mathrm{rpm}$, at $4^{\circ} \mathrm{C}$ for $15 \mathrm{~min}$.

The cell pellet from a $3 \mathrm{~L}$ culture was resuspended in $240 \mathrm{ml}$ lysis buffer. The lysis buffer containing $50 \mathrm{mM}$ Tris-Hcl, pH 8.5, $300 \mathrm{mM} \mathrm{NaCl}, 5 \mathrm{mM}$ benzamidine, $0.1 \mathrm{mM}$ PMSF, $1 \mu \mathrm{g} / \mathrm{ml}$ pepstatin A and $1 \mathrm{mM} \beta$-Me was supplemented with five tablets of protease inhibitor cocktail (Roche, Nutley, NJ) and $0.5 \mathrm{mg} / \mathrm{ml}$ lysozyme (Sigma, St. Loius, MO), $24 \mathrm{ml}$ Bugbuster (Novagen, San Diego, CA), and $6 \mathrm{mM}$ iodoacetamide. Forty micro litres of the Benzonase (Novagen, San Diego, CA) was added to lysis buffer to digest the DNA and reduce the viscosity of the lysate.

\subsubsection{Solubilisation}

The bacterial suspension was incubated for $30 \mathrm{~min}$ at room temperature and centrifuged at 20,000 rpm for $30 \mathrm{~min}$. Since almost all of the BAHC goes into inclusion bodies, the supernatant was discarded. In preliminary experiments, several detergents and sulfobetaine were tried to solubilise the target protein (Vuillard et al., 1998). However, only LSN appeared to be useful (Table 1, Figure 1). In view of that the pellet was resolubilised with $1 \%$ LSN (Fluka, USA) in buffer (50 mM Tris-HCl, pH 8.5, $0.3 \mathrm{M}$ $\mathrm{NaCl}$ and $1 \mathrm{mM} \beta-\mathrm{Me}$ ) and the suspension was kept at room temperature for $2 \mathrm{~h}$ and centrifuged at 20,000 rpm for $30 \mathrm{~min}$ to remove the insoluble material. The supernatant obtained from the above step was allowed to mix with $5 \mathrm{ml}$ Ni-NTA agarose, pre-equilibrated with Tris-HCl buffer ( $50 \mathrm{mM}$ Tris, $\mathrm{pH}$ 8.5, $300 \mathrm{mM} \mathrm{NaCl}, 0.2 \% \mathrm{LSN}$ and $1 \mathrm{mM} \beta$-Me ) at $4^{\circ} \mathrm{C}$ for an hour.

\subsubsection{Purification}

The mixture of Ni-NTA agarose and supernatant was poured into a glass column and the flow-through of the soluble fraction was collected. The column was washed with $100 \mathrm{ml}$ of Tris buffer $+0.1 \%$ LSN followed by $100 \mathrm{ml}$ wash with buffer containing 5, 10 and $20 \mathrm{mM}$ imidazole. Strongly bound protein was eluted with $10 \mathrm{ml}$ of $250 \mathrm{mM}$ (thrice) and $10 \mathrm{ml}$ of $500 \mathrm{mM}$ (twice) imidazole (pH 8.5). Aliquots of all the above fractions were analysed by electrophoresis on $4 \%$ to $20 \%$ Tris-Glycine SDS-PAGE gel followed by staining with Coomassie blue. A $98 \mathrm{kDa}$ band corresponding to BAHC reproducibly eluted in $250 \mathrm{mM}$ imidazole $+0.1 \%$ LSN fractions was obtained (Figure 2). 
Table 1 Detergents and non-detergent sulfobetamine tried for solubilisation

\begin{tabular}{lcc}
\hline Detergent/sulfobetaine & \% used & Solubilisation success (yes/no) \\
\hline Deoxycholate & 1 & No \\
Nonaethylene glycol monoethyl ether & 1 & No \\
Octyl $\beta$-D-glucopyranoside (OG) & 1 & No \\
N-Lauroylsarcosine Sodium salt (LSN) & 1 & Yes \\
Lauryldimethylamine-oxide (LDAO) & 1 & No \\
N,N-Dimethyldecylamine-N-oxide (DDAO) & 1 & No \\
Myristyltrimethylammonium bromide (MTAB) & 1 & No \\
n-dodecyl-beta-D-maltoside (DDM) & 1 & No \\
Dimethylethyl-(3-Sulfopropyl)-ammonium & 15 & No \\
Dimethyl(2-hydroxyethyl)ammonium propane & 15 & No \\
sulfonate & & \\
3-(N-Phenylmethyl-N,N-dimethylammonio) & 15 & No \\
propanesulfonate & & No \\
1-(3-Sulfopropyl)pyridinium betain & 15 & No \\
3-(1-Methylpyridinium)-1-propane sulfonate & 15 & \\
\hline
\end{tabular}

Figure 1 Detergent solubilisation of BAHC

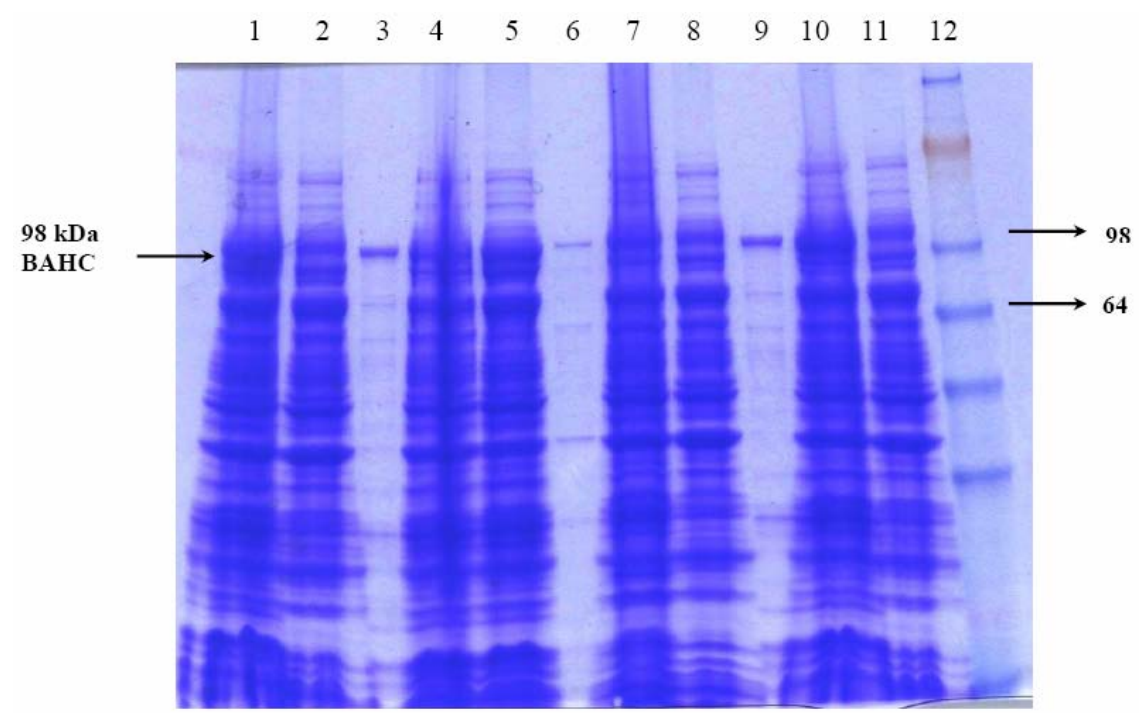

Notes: The samples were run on a $4 \%$ to $20 \%$ Tris-glycine SDS-PAGE gel. Lanes 1-3 (solubilisation with DDM), total lysate, supernatant and pellet; similarly lanes 4-6 (solubilisation with LSN), lanes 7-9 (solubilisation with DDAO), lanes 10 and 11 (solubilisation with OG), and lane 12 molecular weight marker. Though many detergents were tried, only four of them are shown here. While a portion of the protein still remains in pellet (lanes 3 and 9), at least most of it appears in supernatant with LSN (lane 6). The pellet of OG solubilised is not shown. Compared to other detergents and non-detergent sulfobetamine, LSN works the best. 
Figure 2 Overexpression and Ni-NTA purification of BAHC

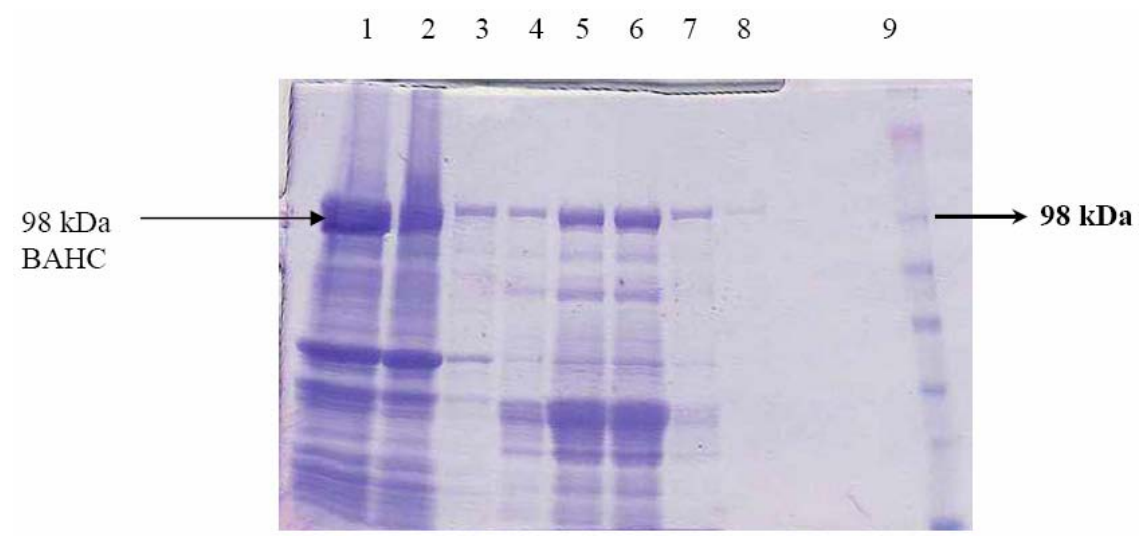

Notes: The samples were run on a $4 \%$ to $20 \%$ Tris-glycine SDS-PAGE gel. Lane 1.

Supernatant after cell lysis of overnight auto-induced cells; lane 2. flow through from Ni-NTA column; lanes 3-8: 20, 50, 250, 250, 250, 500 mM imidazole elute fractions from Ni-NTA column; lane 9: molecular weight marker.

Figure 3 The size exclusion profile of the BAHC

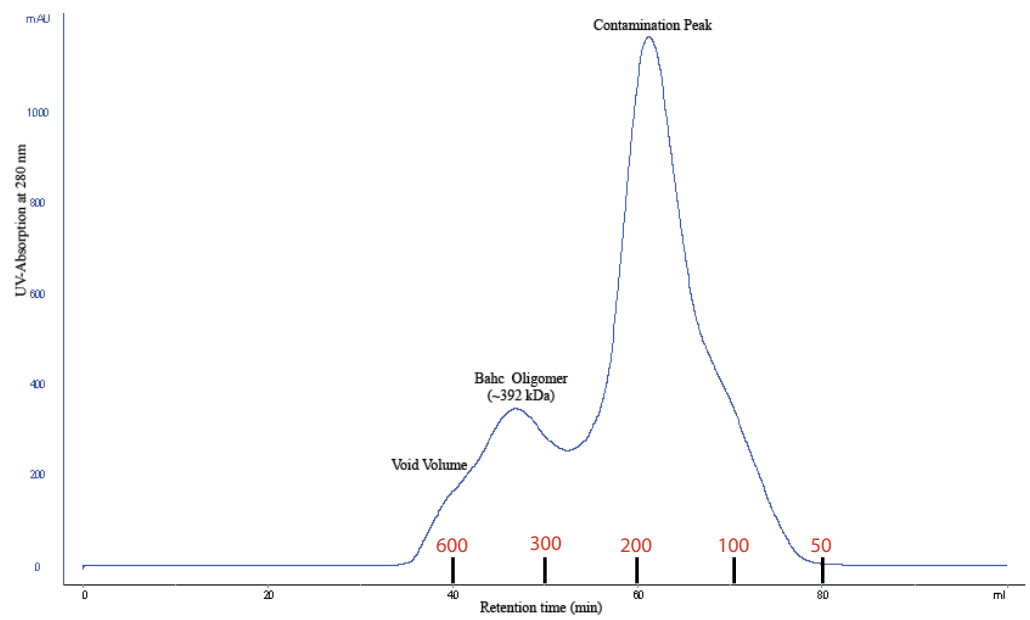

Notes: $5 \mathrm{ml}$ of Ni-affinity purified sample was loaded into a preparative S300 column. Protein sizes are shown in red colour along the X-axis. BAHC-LSN oligomer has an apparent molecular mass of $\sim 392 \mathrm{kDa}$ corresponding to a tetramer of BAHC (98 kDa).

The protein was further purified by a size-exclusion (Sephacryl-300) column that had previously been equilibrated with $50 \mathrm{mM}$ Tris, $\mathrm{pH} 8.5,300 \mathrm{mM} \mathrm{NaCl}, 0.1 \% \mathrm{LSN}$ and $1 \mathrm{mM} \beta$-Me. The elution profile, shown in Figure 3 indicates that the protein eluted as tetramer. Highly purified protein obtained after the size exclusion column is shown in SDS-PAGE (Figure 4). Three litres of auto-induction medium yielded about $2 \mathrm{mg}$ protein 
with $>90 \%$ purity. Western blot using polyclonal rabbit IgG of BoNT/A confirmed the protein is BAHC.

Figure 4 Gel-filtration chromatography of BAHC

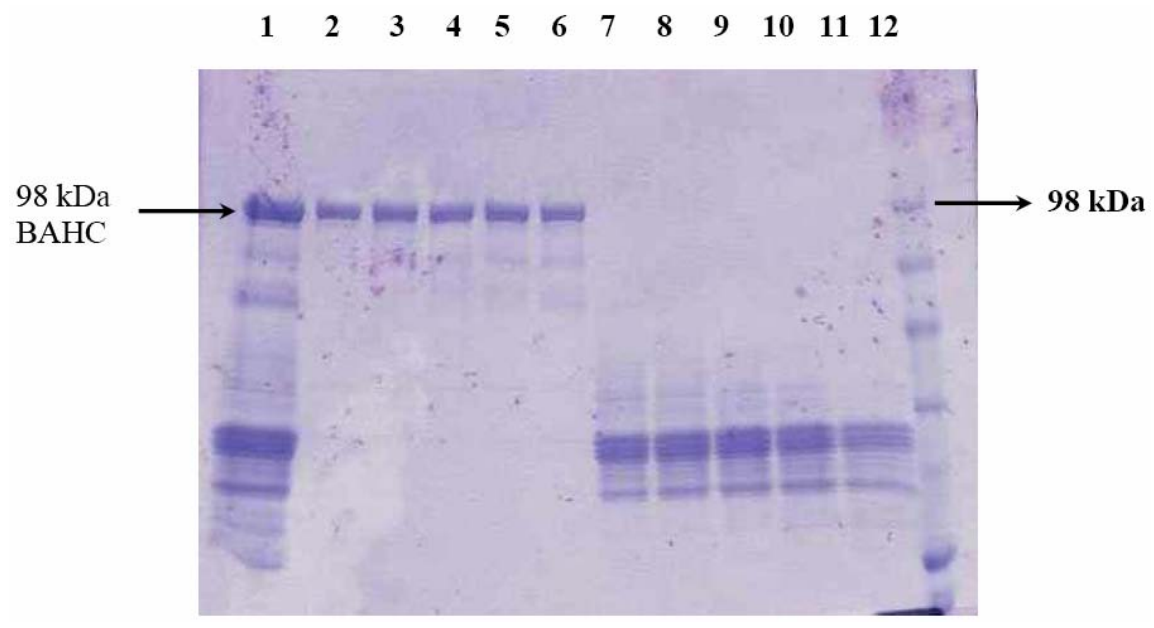

Notes: The samples were run on a $4 \%$ to $20 \%$ Tris-glycine SDS-PAGE gel. Lane 1 . pooled fractions of Ni-NTA column before size-exclusion chromatography, lanes 2-6, fractions of BAHC, lanes 7-11, fractions of contamination, lane 12 molecular weight marker.

\subsubsection{Western blot analysis}

Extracted BAHC proteins after his-tag purification were separated by SDS-PAGE on $4 \%$ to $20 \%$ gradient polyacrylamide gels and electrophoretically transferred onto Nitrocellulose membranes (Figure 5). The membranes were probed with BAHC primary antibody raised against rabbit, followed by incubation with horseradish peroxidase-coupled secondary antibody. Detection was performed with a chemiluminescence-based detection kit.

\subsection{Crystallisation and preliminary crystallographic data analysis}

Crystals were grown at room temperature with 20\% PEG 3350, 0.2M Tri-sodium citrate as precipitant $\left(1 \mu \mathrm{l}\right.$ reservoir solution plus $1 \mu \mathrm{l}$ protein at $5 \mathrm{mg} \mathrm{ml}^{-1}$ in $50 \mathrm{mM}$ Tris-HCl $\mathrm{pH}$ 8.5, $300 \mathrm{mM} \mathrm{NaCl}, 0.1 \% \mathrm{LSN}$ and $1 \mathrm{mM} \beta-\mathrm{Me}$ ) using the sitting drop vapour diffusion technique. Crystals appeared within 15 days. Crystals were very briefly soaked in the mother liquor containing 15\% glycerol added as cryoprotectant and immediately flash frozen in liquid nitrogen. X-ray diffraction data were collected at beamline X29 of NSLS (National Synchrotron Light Source, Brookhaven National Laboratory, New York) and were processed using HKL2000 (Otwinowski and Minor, 1997). The diffraction quality was both poor (maximum resolution $\sim 6 \AA$ ) and anisotropic. 
Figure 5 Western blot analysis of BAHC after Ni-NTA affinity chromatography

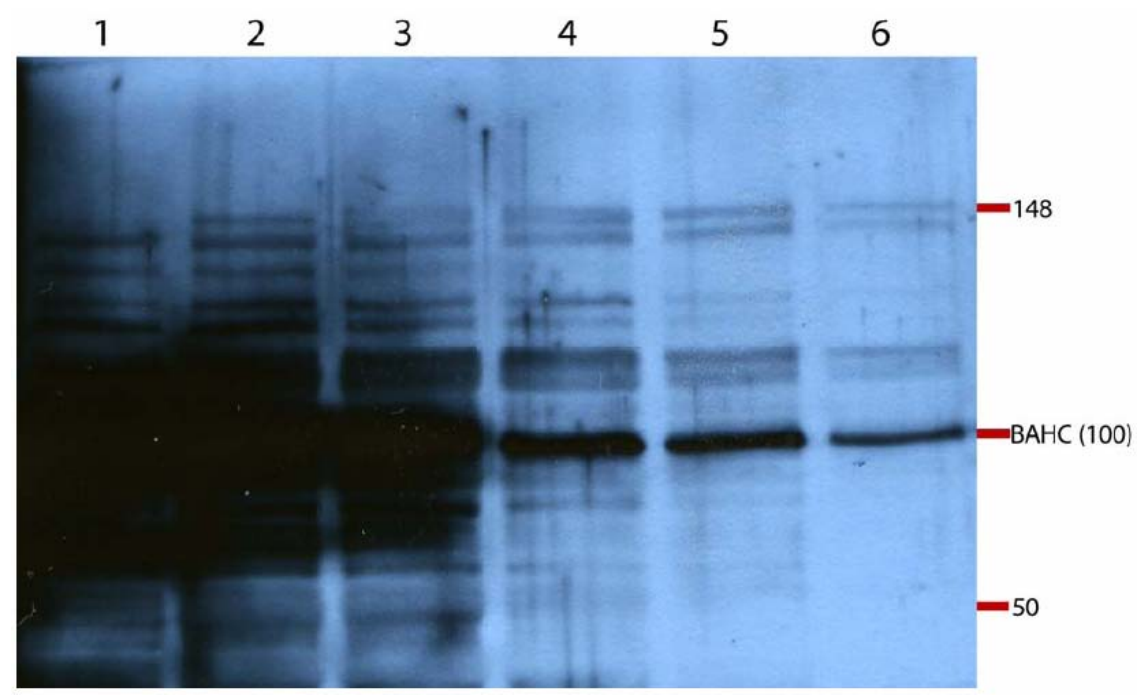

Note: Lanes 1 to 6 corresponds to $20 \mathrm{mM}, 50 \mathrm{mM}, 100 \mathrm{mM}$ elution 1, $100 \mathrm{mM}$ elution 2, $250 \mathrm{mM}$ and $500 \mathrm{mM}$ concentration of imidazole respectively.

\section{Results and discussion}

The BAHC contains residues from Ala448 to Leu1295 with a C-terminal 6X-His tag to enable purification by metal affinity chromatography. However, in the absence of a detergent, it forms inclusion bodies presumably due to the hydrophobic nature of the translocation domain. Similar results were obtained when auto induction was carried out at 20 and $37^{\circ} \mathrm{C}$. Variation in cell growth incubation temperature, time and shaker speed did not yield the soluble protein. Expression of BAHC using conventional IPTG induction method did not help either. Initial screening of solubilsation was performed with 13 different detergents/sulfobetaines that include mild non-ionic detergents such as n-Dodecyl- $\beta$-D-maltoside (DDM) and n-Octyl- $\beta$-D-glucopyronoside (OG) (Table 1). Only LSN gave soluble protein and accordingly $0.1 \%$ LSN was used in buffers throughout BAHC purification process including the initial step of Ni-NTA affinity chromatography. Size exclusion chromatography shows that BAHC is eluted from the NTA resin as tetramer according to elution profile relative to known mol. wt. standards (Figure 3).

Different attempts were made to reduce the detergent concentration from the sample buffer prior to crystallisation but ended up in precipitation. Therefore, BAHC fractions collected from the size exclusion chromatography is used as such in the same LSN concentration for further crystallisation trials. The purified BAHC was crystallised by the sitting drop vapour diffusion method (Figure 6). The denaturation followed by refolding experiment on BAHC was not successful in terms of getting crystals and hence it is not discussed here. Crystals of BAHC diffracted only up to $\sim 6 \AA$, belong to the C-centred orthorhombic space group and contain four molecules in the asymmetric unit. A representative diffraction pattern is shown in Figure 7 and data statistics are given in 
Table 2. A self-rotation Patterson map was computed using MOLREP (Ccp4, 1994) which confirmed the presence of a non-crystallographic four-fold symmetry (Figure 8) consistent with tetrameric association seen in the sizing profile.

Figure 6 Representative crystals of the BAHC

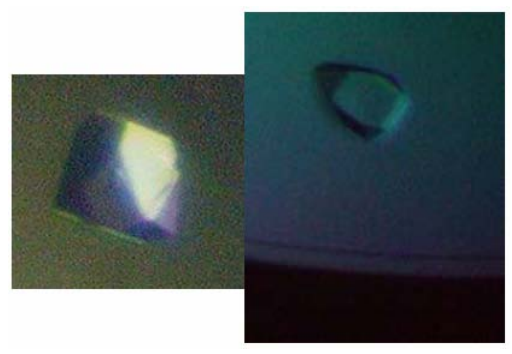

Figure 7 Diffraction pattern from the recombinant BAHC crystal

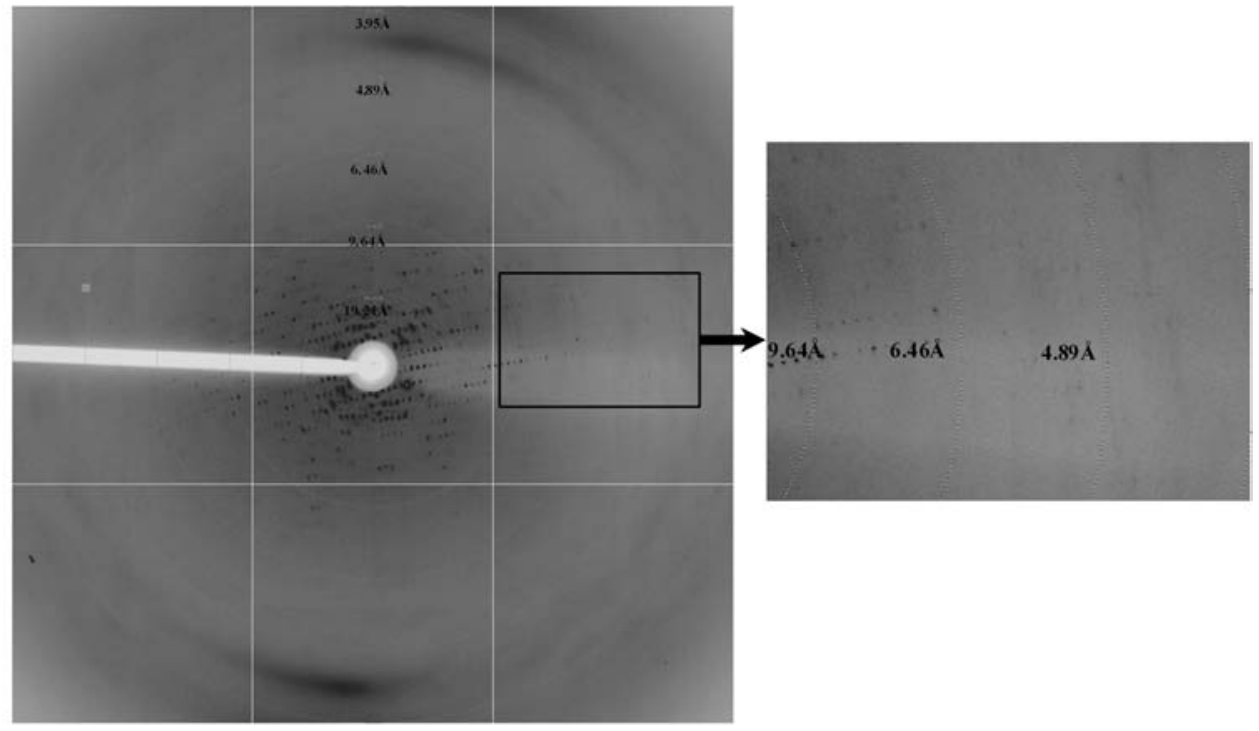

Note: The crystals diffract to better than $6.0 \AA$ resolution. The inset shows the extent of the resolution limit.

Table 2 Data collection statistics of recombinant BAHC

\begin{tabular}{ll}
\hline Cell parameters & $\mathrm{a}=176.33, \mathrm{~b}=225.35$ and $\mathrm{c}=218.20 \AA$ \\
Space group & $\mathrm{C} 222$ \\
Resolution & $50-6.0 \AA$ \\
No. of reflection & 32,674 \\
$\mathrm{I} / \sigma(\mathrm{I})$ & 6.4 \\
$\mathrm{R}_{\text {merge }}$ & $0.08(0.41)$ \\
Redundancy & 3.0 \\
\hline
\end{tabular}


Figure $8 \chi=90^{\circ}$ section of the stereographic projection of Patterson self-rotation function calculated with reflections in the resolution range $50-6.0 \AA$

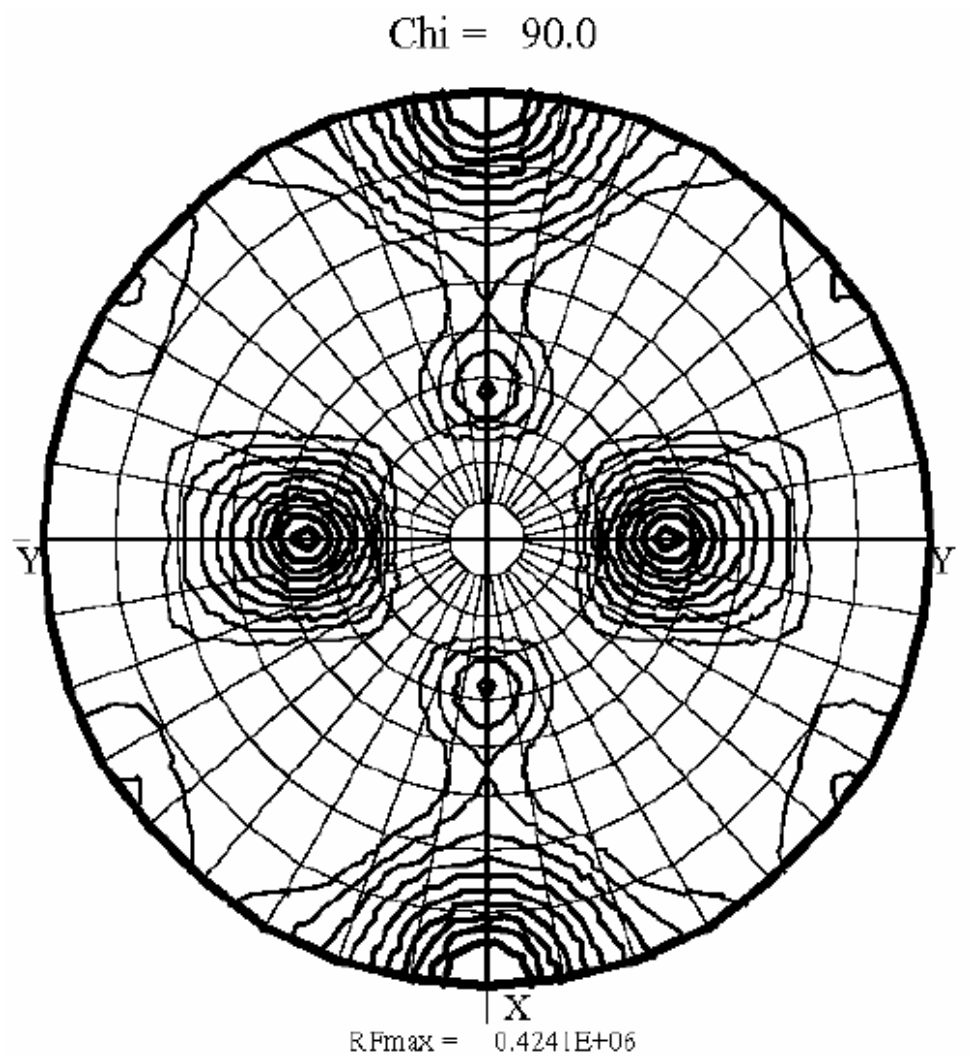

Note: The non-crystallographic four-fold axis is seen at $\theta=45^{\circ}$ and $\phi=90^{\circ}$.

BoNT HC channels in lipid bilayers have been studied for the last three decades (Blaustein et al., 1987; Donovan and Middlebrook, 1986; Hoch et al., 1985; Lebeda and Singh, 1999). Electron microscopy studies in the presence of phospholipid bilayers suggest that BoNT oligomerises to a tetrameric state to form a LC conducting channel (Schmid et al., 1993). Similarly, BoNT/C also forms a channel with two or more monomers (Donovan and Middlebrook, 1986). The initial and the final steps of the translocation process are known but the actual translocation process remains unexplained.

Crystal structures of intact BoNT/A, BoNT/B and BoNT/E are available. BoNT/E differs in domain organisation from BoNT/A and BoNT/B though all of them share significant sequence homology. BoNT/E has inherent translocation-competent domain organisation which causes faster action compared to BoNT/A (Kumaran et al., 2009). BoNT/A or /B has to be reoriented in the vesicle about the binding domain for it to attain a translocation competent conformation (Fu et al., 2009). It is also suggested that the translocation and catalytic domains might rotate about a flexible hinge region between the binding and translocation domains (Fu et al., 2009; Kumaran et al., 2009) to bring the transmembrane region of the translocation domain close to the vesicle membrane. Hence, there is a possibility for the presence of intrinsic domain flexibility which might be an impediment for the formation of good diffracting quality crystals of the HC domain 
alone. This idea is consistent with electron microscopy studies on intact BoNT/E which show the presence of at least three types of domain organisation (Fu et al., 2009). A ribbons representation of BoNT/A is shown in Figure 9(a). The belt region which is part of the translocation domain wraps around the LC and acts as an intramolecular chaperone (Brunger et al., 2007) and without this belt the translocation domain has higher tendency to aggregate (Galloux et al., 2008). When the LC is deleted from the construct the belt region and one side of the translocation domain lose the hydrophobic contacts provided by the LC [Figure 9(b)]. Also, the belt region forming a long loop becomes flexible and can take various conformations. Because of this and the exposure of hydrophobic core, the protein tends to aggregate and probably is the reason for it going into inclusion bodies. The translocation domain of BoNT/A binds to anionic membrane and then permeates the bilayer without any detectable structural change observed by vesicle permeabilisation assays and also the belt region limits protein-membrane interaction (Galloux et al., 2008). In the absence of the LC, the hydrophobic regions of the translocation domain including the belt region are exposed causing aggregation and the use of detergents helps to avoid this problem. The flexibility of the belt region also may be partially compensated by the HC oligomerisation.

Figure 9 (a) Ribbons representation of BoNT/A molecule* (b) BAHC alone is shown**

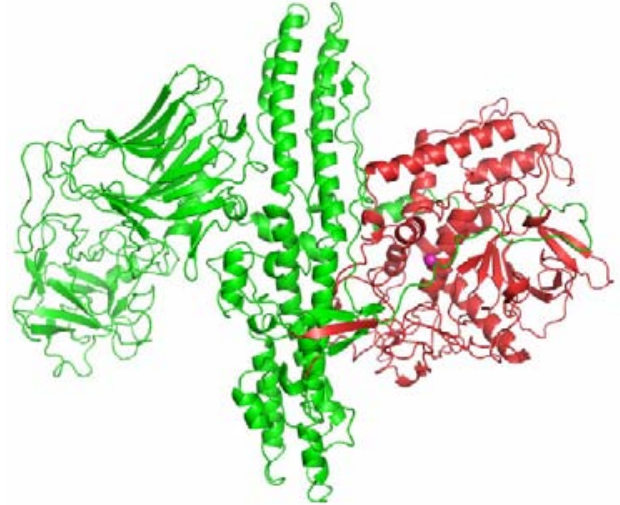

(a)

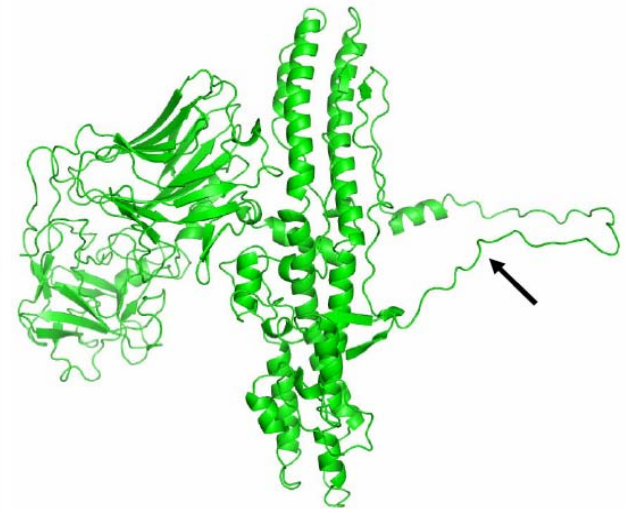

(b)

Notes: *This figure was created using PDB id: 3BTA and was drawn with Pymol (Delano, 2002). The HC, BAHC, is shown in green and the LC is shown in red. Zinc ion at the active site is shown as magenta sphere. The belt region wrapping around the LC is obvious in this figure. **When the LC is removed the belt region (shown by arrow mark) becomes very flexible since it loses all its interaction with the LC.

While crystallising the HC alone, the inherent flexibility of binding domain and the belt region might cause disorder in the crystal lattice; the presence of detergent mimicking a membrane environment may also be responsible for poor diffraction quality crystals. BoNT/A protein is suggested to make a channel immediately after the membrane insertion (Fischer and Montal, 2007b). Accordingly, the channel or oligomerisation state of BAHC might have occurred because of the use of detergent from the initial step of protein extraction. 


\section{Conclusions}

The HC of BoNT/A was solubilised with detergent LSN and its gel filtration column chromatography showed that the oligomeric state is a tetramer. A self-rotation plot from the X-ray diffraction data shows a four-fold symmetry in the crystal structure. This is the first crystallographic study showing support for tetrameric pore formation by the BAHC for LC translocation. In this study, the oligomeric state was obtained in the presence of detergent that partly mimics the channel formation at endosomal membrane although at different $\mathrm{pH}$. Further high resolution crystal structure might reveal the pore formation and help in understanding the translocation mechanism.

\section{Acknowledgements}

We gratefully acknowledge the financial support from Allergan, Inc., USA, and data collection support from beamline X29 (NSLS).

\section{References}

Blaustein, R.O., Germann, W.J., Finkelstein, A. and Dasgupta, B.R. (1987) 'The N-terminal half of the heavy chain of botulinum type A neurotoxin forms channels in planar phospholipid bilayers', FEBS Lett., Vol. 226, No. 1, pp.115-120.

Brunger, A.T., Breidenbach, M.A., Jin, R., Fischer, A., Santos, J.S. and Montal, M. (2007) 'Botulinum neurotoxin heavy chain belt as an intramolecular chaperone for the light chain', PLoS Pathog., Vol. 3, No. 9, pp.1191-1194.

Ccp4 (1994) 'CCP4 suite: programs for protein crystallography’, Acta Crystallogr., Vol. D50, Part 5, pp.760-763.

Chai, Q., Arndt, J.W., Dong, M., Tepp, W.H., Johnson, E.A., Chapmann, E.R. and Stevens, R.C. (2006) 'Structural basis of cell surface receptor recognition by botulinum neurotoxin B', Nature (London), New Biology, Vol. 444, pp.1019-1020.

Delano, W.L. (2002) 'Pymol', The PyMOL User's Manual DeLano Scientific, San Carlos, CA, USA.

Donovan, J.J. and Middlebrook, J.L. (1986) 'Ion-conducting channels produced by botulinum toxin in planar lipid membranes’, Biochemistry and Cell Biology, Vol. 25, No. 10, pp.2872-2876.

Fischer, A. and Montal, M. (2007a) 'Crucial role of the disulfide bridge between botulinum neurotoxin light and heavy chains in protease translocation across membranes', J. Biol. Chem., Vol. 282, No. 40, pp.29604-29611.

Fischer, A. and Montal, M. (2007b) 'Single molecule detection of intermediates during botulinum neurotoxin translocation across membranes', Proc. Natl. Acad. Sci., USA, Vol. 104, pp.10447-10452.

Fischer, A., Mushrush, D.J., Lacy, D.B. and Montal, M. (2008) 'Botulinum neurotoxin devoid of receptor binding domain translocates active protease’, PLoS Pathog., Vol. 4, No. 12, p.e1000245.

Fischer, A., Nakai, Y., Eubanks, L.M., Clancy, C.M., Tepp, W.H., Pellett, S., Dickerson, T.J., Johnson, E.A., Janda, K.D. and Montal, M. (2009) 'Bimodal modulation of the botulinum neurotoxin protein-conducting channel', Proc. Natl. Acad. Sci. USA, Vol. 106, No. 5, pp.1330-1335.

Fu, Z., Chen, C., Barbieri, J.T., Kim, J.J. and Baldwin, M.R. (2009) 'Glycosylated SV2 and gangliosides as dual receptors for botulinum neurotoxin serotype F', Biochemistry, Vol. 48, No. 24, pp.5631-5641. 
Galloux, M., Vitrac, H., Montagner, C., Raffestin, S., Popoff, M.R., Chenal, A., Forge, V. and Gillet, D. (2008) 'Membrane interaction of botulinum neurotoxin A translocation (T) domain. The belt region is a regulatory loop for membrane interaction', J. Biol. Chem., Vol. 283, No. 41, pp.27668-27676.

Hoch, D.H., Romero-Mira, M., Ehrlich, B.E., Finkelstein, A., Dasgupta, B.R. and Simpson, L.L. (1985) 'Channels formed by botulinum, tetanus, and diphtheria toxins in planar lipid bilayers: relevance to translocation of proteins across membranes', Proc. Natl. Acad. Sci., USA, Vol. 82, No. 6, pp.1692-1696.

Koriazova, L. and Montal, M. (2003) 'Translocation of botulinum neurotoxin light chain protease through the heavy chain channel', Nat. Struct. Biol., Vol. 10, No. 1, pp.13-18.

Kumaran, D., Eswaramoorthy, S., Furey, W., Navaza, J., Sax, M. and Swaminathan, S. (2009) 'Domain organization in Clostridium botulinum neurotoxin type $\mathrm{E}$ is unique: its implication in faster translocation', J. Mol. Biol., Vol. 386, No. 1, pp.233-245.

Kumaran, D., Rawat, R., Ahmed, S.A. and Swaminathan, S. (2008) 'Substrate binding mode and its implication on drug design for botulinum neurotoxin A', PLoS Pathog., Vol. 4, No. 9, p.e1000165.

Lacy, D.B. and Stevens, R.C. (1999) 'Sequence homology and structural analysis of clostridial neurotoxins’, J. Mol. Biol., Vol. 291, No. 5, pp.1091-1104.

Lacy, D.B., Tepp, W., Cohen, A.C., Dasgupta, B.R. and Stevens, R.C. (1998) 'Crystal structure of botulinum neurotoxin type A and implications for toxicity’, Nat. Struct. Biol., Vol. 5, No. 10, pp.898-902.

Lebeda, F.J. and Singh, B.R. (1999) 'Membrane channel activity and translocation of tetanus and botulinum neurotoxins', Toxin Reviews, Vol. 18, pp.45-76.

Li, L. and Singh, B.R. (1999) 'In vitro translation of type A Clostridium botulinum neurotoxin heavy chain and analysis of its binding to rat synaptosomes', J. Prot. Chem., Vol. 18, No. 1, pp.89-95.

Montal, M. (2009) 'Translocation of botulinum neurotoxin light chain protease by the heavy chain protein-conducting channel', Toxicon, Vol. 54, No. 5, pp.565-569.

Montal, M., Montal, M.S. and Tomich, J.M. (1990) 'Synporins - synthetic proteins that emulate the pore structure of biological ionic channels', Proc. Natl. Acad. Sci., USA, Vol. 87, No. 18, pp.6929-6933.

Montecucco, C. (1986) 'How do tetanus and botulinum toxins bind to neuronal membranes?', Trends in Biochemical Science, Vol. 11, pp.314-317.

Otwinowski, Z. and Minor, W. (1997) 'Processing of X-ray diffraction data collected in oscillation mode', Methods Enzymol., Vol. 276, pp.307-326.

Parikh, S. and Singh, B.R. (2007) 'Comparative membrane channel size and activity of botulinum neurotoxins A and E', Protein J., Vol. 26, No. 1, pp.19-28.

Pless, D.D., Torres, E.R., Reinke, E.K. and Bavari, S. (2001) 'High-affinity, protective antibodies to the binding domain of botulinum neurotoxin type A', Infect. Immun., Vol. 69, No. 1, pp.570-574.

Schmid, M.F., Robinson, J.P. and Dasgupta, B.R. (1993) 'Direct visualization of botulinum neurotoxin induced channels in phospholipid vesicles', Nature, Vol. 364, No. 6440, pp.827-830.

Segelke, B., Knapp, M., Kadkhodayan, S., Balhorn, R. and Rupp, B. (2004) 'Crystal structure of Clostridium botulinum neurotoxin protease in a product-bound state: evidence for noncanonical zinc protease activity', Proc. Natl. Acad. Sci. USA, Vol. 101, No. 18, pp.6888-6893.

Sheridan, R.E. (1998) 'Gating and permeability of ion channels produced by botulinum toxin types A and E in PC12 cell membranes’, Toxicon, Vol. 36, pp.703-717.

Simpson, L.L. (1986) 'Molecular pharmacology of botulinum toxin and tetanus toxin', Annu. Rev. Pharmacol. Toxicol., Vol. 26, pp.427-453. 
Simpson, L.L. (Ed.) (1989) Botulinum Neurotoxins and Tetanus Toxin, Academic Press, New York.

Singh, B.R., Barcomb-Caddle, L.A., Fu, F.N. and Li, B. (1996) 'Gene probe-based detection of type $\mathrm{E}$ botulinum neurotoxin binding protein using polymerase chain reaction', Toxicon, Vol. 34, No. 7, pp.737-742.

Stenmark, P., Dupuy, J., Imamura, A., Kiso, M. and Stevens, R.C. (2008) 'Crystal structure of botulinum neurotoxin type A in complex with the cell surface co-receptor GT1b-insight into the toxin-neuron interaction', PLoS Pathog., Vol. 4, No. 8, p.e1000129.

Studier, F.W. (2005) 'Protein production by auto-induction in high-density shaking cultures', Protein Expr. Purif., Vol. 41, No. 1, pp.207-234.

Szabo, E.A., Pemberton, J.M. and Desmarchelier, P.M. (1993) 'Detection of the genes encoding botulinum neurotoxin types A to $\mathrm{E}$ by the polymerase chain reaction', App. Environ. Microbiol., Vol. 59, No. 9, pp.3011-3020.

Vuillard, L., Rabilloud, T. and Goldberg, M.E. (1998) 'Interactions of non-detergent sulfobetaines with early folding intermediates facilitate in vitro protein renaturation', Eur. J. Biochem., Vol. 256, No. 1, pp.128-135.

Yowler, B.C., Kensinger, R.D. and Schengrund, C.L. (2002) 'Botulinum neurotoxin A activity is dependent upon the presence of specific gangliosides in neuroblastoma cells expressing synaptotagmin I’, J. Biol. Chem., Vol. 277, No. 36, pp.32815-32819. 